\title{
A head-to-head comparison of wall motion score index, force, strain, and ejection fraction for the prediction of SYNTAX and Gensini coronary scores by dobutamine stress echocardiography
}

\author{
Karina Wierzbowska-Drabik'1, Eugenio Picano², Michał Simiera1', Michał Plewka1, \\ Radosław Kręcki', Jan Z. Peruga', Jarosław D. Kasprzak \\ 1 1st Department and Chair of Cardiology, Medical University of Lodz, Biegański Hospital, Łódź, Poland \\ 2 Institute of Clinical Physiology, National Council Research, Pisa, Italy
}

\section{KEY WORDS}

force, strain, stress echocardiography, SYNTAX score, wall motion index
Correspondence to: Karina Wierzbowska-Drabik, MD, PhD, 1st Department and Chair of Cardiology, Medical University of Lodz, ul. Kniaziewicza 1/5, 91-347 tódź, Poland, phone: +48426539909, email: wierzbowska@ptkardio.pl Received: March 31, 2020. Revision accepted: May 12, 2020. Published online: May 19, 2020. Kardiol Pol. 2020; 78 (7-8): 715-724 doi:10.33963/KP.15376 Copyright by the Author(s), 2020

\begin{abstract}
BACKGROUND Predicting the severity of coronary artery disease (CAD) may be possible during dobutamine stress echocardiography (DSE) with various indices of left ventricular function.

AIMS We assessed the relative value of ejection fraction (EF), force, global longitudinal strain (GLS), and wall motion score index (WMSI) in predicting SYNTAX and Gensini scores in patients with known or suspected CAD.

METHODS We prospectively enrolled 223 patients (120 men; mean [SD] age, 62 [9] years) and assessed the following indices: 1) EF (with triplane imaging); 2) force, calculated as the ratio of systolic blood pressure to left ventricular end-systolic volume; 3) GLS; 4) WMSI. All patients underwent coronary angiography within 12 weeks with SYNTAX and Gensini scores evaluation.

RESULTS The correlation of SYNTAX and Gensini scores was highest with peak WMSI (SYNTAX, rho $=0.591$; Gensini, rho $=0.612 ; P<0.001)$, intermediate with peak force (SYNTAX, rho $=-0.346$; Gensini, rho $=-0.377$; $P<0.001$ ) and GLS (SYNTAX, rho $=-0.205 ; P=0.002$ and Gensini rho $=-0.216 ; P=0.001)$, and the weakest for EF (SYNTAX, rho $=-0.149 ; P=0.03$ and Gensini, rho $=-0.191 ; P=0.006)$. The similar hierarchy of variables was detected for changes during DSE. In the subgroup after myocardial infarction $(n=66[27 \%])$, GLS outperformed the force.

CONCLUSIONS In patients with CAD at DSE peak, the WMSI and force were better predictors of the coronary SYNTAX and Gensini scores and CAD severity than GLS or EF. However, in patients after myocardial infarction, the GLS correlation with coronary scores improved and got closer to the visual assessment.
\end{abstract}

INTRODUCTION SYNTAX (Synergy between Percutaneous Coronary Intervention with TAXUS and Cardiac Surgery) ${ }^{1}$ and Gensini ${ }^{2}$ scores quantify the coronary artery disease (CAD) with a prognostic value documented in various clinical scenarios. ${ }^{3-6}$ The extent and severity of CAD are mirrored by the degree of left ventricular (LV) dysfunction during stress echocardiography (SE) 7,8 and represent critically important information for risk stratification and revascularization. ${ }^{9}$ The wall motion score index (WMSI) is by far the most used and validated index in SE. ${ }^{10,11}$ The ejection fraction (EF) is a recognized hallmark for prognosis in clinical cardiology included in the minimal data set of SE. ${ }^{12}$ With advanced imaging, global longitudinal strain (GLS) provides a quantitative parameter of the LV function, which can be impaired when EF is normal. ${ }^{13}$ The LV force, also known as elastance, ${ }^{14}$ is calculated as the ratio of systolic blood pressure 


\section{WHAT'S NEW?}

Dobutamine stress echocardiography (DSE) is a test recommended for the diagnosis of coronary artery disease (CAD), which may be also helpful in assessing its severity. SYNTAX and Gensini scores show the severity of CAD. Our aim was to assess which of the indices indicating left ventricular function used during DSE (wall motion score index [WMSI], ejection fraction [EF], force, or global longitudinal strain [GLS]) reflects the best SYNTAX and Gensini scores in patients with suspected or known CAD. The analysis of 223 patients enabled us to rank proposed indices from the most to least related with CAD severity: 1) WMSI, 2) force and GLS, 3) ejection fraction. Such an analysis has not been performed in one patient group so far. Furthermore, we observed that in patients after myocardial infarction, the GLS correlation with coronary scores improved, which has practical implication for integrated use of these parameters during DSE. measured LV end-systolic volume. Echocardiographic measurements were made with accordance to guidelines. ${ }^{21}$ The evaluation of regional contractility was performed by 2 cardiologists (KWD and JDK) and each segment was classified from normokinetic to dyskinetic, with 1 to 4 points given respectively. WMSI was calculated at rest and during peak stress by adding the points and dividing them by the number of segments. The worsening of contractility in at least 2 adjacent segments of the LV was consistent with a positive DSE. Dobutamine was administered by intravenous infusion in doses of $10,20,30$, and $40 \mu \mathrm{g} / \mathrm{kg} / \mathrm{min}$ during 3-minute stages, and atropine was added in 0.5 -mg fractional doses after the second stage of infusion, up to $2 \mathrm{mg}$. The infusion of dobutamine was stopped when the heart rate (HR) limit, positive test, or other criteria of ending the examination were fulfilled. ${ }^{22,23}$ vides a load-independent assessment of LV contractility, ${ }^{15,16}$ which is more efficient than EF in predicting the outcome in negative $\mathrm{SE} .^{17,18}$

Our aim was to provide a head-to-head comparison of 4 indices of the LV systolic function (EF, GLS, force, and WMSI) at rest and during peak DSE for the prediction of CAD extent and severity expressed as SYNTAX and Gensini scores taking also into account the presence of previous myocardial infarction (MI).

METHODS Study group and protocol We prospectively included 223 patients with stable angina who underwent DSE with an early atropine administration and then had coronary angiography performed within 3 months. Inclusion criteria were: 1 ) rest and DSE study of acceptable quality; 2) sinus rhythm; 3) informed consent to enter the study; 4) angiography with a calculation of SYNTAX and Gensini scores. Exclusion criteria were: 1) severe valve disease or atrial fibrillation; 2) contraindications to atropine or dobutamine. ${ }^{19,20}$

All patients gave written informed consent to participate in the study and the protocol was approved by the ethics committee of the Medical University of Lodz (no. RNN/119/10 KE; July 13, 2010).

Echocardiography at rest and during dobutamine stress echocardiography Transthoracic echocardiography was performed with the VIVID 7 device (GE Vingmed Ultrasound AS, Horten, Norway) using the M4S probe in harmonic mode operating at 2.0/4.3 $\mathrm{MHz}$ with the maximal frame per second count available at the necessary sector width. The range of frame per second was from 64 to 112, with a mean value of 83 . The assessment of the LV systolic and diastolic volumes and EF was made from the triplane view with a volumetric probe (3V). The LV force was calculated as the ratio of the systolic blood pressure to simultaneously
Assessment of deformation Loops from echocardiographic views were digitally stored. Deformation parameters were calculated on the EchoPac 6.1.0 workstation (GE Vingmed Ultrasound). Three points ( 2 on the basal and 1 on the apical endocardium) were marked in each apical view and the region of interest was accepted to obtain the systolic longitudinal strain by the automated function imaging method. The segmental values of the peak systolic longitudinal strain were displayed as polar maps, with information regarding the global (from 17 segments) systolic longitudinal strain (GLS). To facilitate the interpretation of changes and correlations of GLS data, we used the absolute values of the longitudinal strain.

Assessment of SYNTAX and Gensini scores The Gensini score takes into account the increasing severity of luminal stenosis with $25 \%, 50 \%$, $75 \%, 90 \%, 99 \%$, and $100 \%$ diameter reduction as well as lesion location. ${ }^{2}$ The SYNTAX score was established to grade the anatomical complexity of coronary lesions in patients with left main or 3 -vessel disease. It shows the predictive value for cardiac and cerebrovascular events in patients treated with percutaneous coronary intervention and guides the selection of optimal treatment. The SYNTAX score was calculated using an open access online calculator (www.syntaxscore.com) and the Gensini score was obtained by multiplication of the severity score by the segment location multiplying factor. Both scores were assessed by 2 invasive cardiologists blinded to DSE results (MS and MP).

Statistical analysis Statistical analysis was performed using MedCalc V. 12.1.4. (MedCalc Software Ltd., Ostend, Belgium). Continuous variables were expressed as means (SD), categorical as numbers and percentages. A comparison 
of variables at the baseline and peak stage of DSE in the same group was made with the $t$ test for paired samples. The normal distribution of variables was tested with D'Agostino-Pearson test. Spearman rank correlation coefficients (rho) were calculated to assess the correlation of indices of the global LV function. A $P$ value of less than 0.05 was accepted as significant. Interobserver variability was tested by calculating coefficient of variation (CoV) and weighted kappa for ordinal variables as well as additionally with intraclass correlation coefficient.

RESULTS Feasibility and interobserver variability All LV segments of patients included in the study were feasible for visual assessment since the obtaining of fully readable apical views at consecutive SE stages was the main presupposed focus for the performing cardiologist (at the cost of not including a wider range of parameters into protocol, eg, attempts at Doppler analysis of coronary flow). For volumetric data (end-systolic volume and $\mathrm{EF}$ ), we used a $3 \mathrm{~V}$ probe and triplane method, enabling the acquisition of all apical views at one time point, achieving $100 \%$ feasible measurements at rest and during stress, respectively $92 \%$ for both parameters. For GLS analysis, we based on consecutive 2-dimensional apical views and accepted maximally 2 segments lacking strain for GLS calculation of the left ventricle. As far as the feasibility of regional analysis is concerned, it achieved $99.5 \%$ for rest and $99 \%$ during stress which render a sum of 60 segments ( $0.8 \%$ ) excluded during both stages of DSE for a total of 7582 segments, mainly in the region of the lateral wall. ${ }^{19}$

Interobserver variability for echocardiographic parameters was assessed for the baseline and peak stage of DSE and results were obtained as the average of 3 measurements by each of the observers.

The agreement was very good for WMSI and GLS at both stages (with the $\mathrm{CoV} \leq 10 \%$ ) and lower, but still good, for parameters requiring measurement of the left ventricular volume, that is, $\mathrm{EF}$ and force (with $\mathrm{CoV} \leq 15 \%)$. For the angiographic score, we found acceptable agreement for both scores; however, it was better for the Gensini score with $\mathrm{CoV}=10.4 \%$ and worse for the SYNTAX score with $\mathrm{CoV}=17.8 \%$. The weighted kappa showed very good interobserver agreement for the Gensini score (kappa $=0.840)$ and good for the SYNTAX score (kappa $=0.674$ ). Intraclass correlation coefficients for echocardiographic parameters at stress were: 0.88 for EF, 0.94 for GLS, 0.93 for force, and 0.99 for WMSI.

Clinical, echocardiographic, and angiographic data Demographic and clinical characteristics of the study group are presented in TABLE 1 , showing the significant burden of cardiovascular risk factors and a $30 \%$ prevalence of MI history.
Comparison of hemodynamic and echocardiographic data during DSE is presented in TABLE 2; coronary angiography data are presented in TABLE3.

A total of 186 patients (83\%) achieved the target HR limit during DSE with an early atropine administration and 128 patients $(57 \%)$ showed a positive DSE for inducible wall motion abnormalities. Significant CAD was present in 112 patients (50.2\%), and absent in 111 (49.8\%). We found a very strong correlation between both parameters describing the severity of coronary lesions: the SYNTAX score (ranging from 0 to 50) and the Gensini score (ranging 0 to 128 ) with rho $=0.962(P<0.001)$.

Correlation between left ventricular function parameters and angiographic scores In the whole group of 223 patients, we observed a significant moderate to strong correlation between SYNTAX and Gensini scores and WMSI, which reached the highest coefficient at the peak stress stage (Gensini score, rho $=0.612 ; P<0.001$ and SYNTAX score, rho $=0.591, P<0.001$ ), see TABLE 4 and FIGURE 1 A. WMSI based on a visual assessment of segmental thickening had the highest correlation coefficient (rho $=0.6$; calculated as average value for both scores) and was followed directly by force (rho $=0.4$ ), whereas the correlation of GLS and EF with coronary severity indexes was weaker (rho $=0.2$ for both) (FIGURE 2).

As far as the changes of indices between peak and base DSE stages are concerned $(\Delta)$, severity of CAD correlated moderately with $\triangle \mathrm{WMSI}$ (SYNTAX score, rho $=0.494 ; P<0.001$ and Gensini score, rho $=0.496 ; P<0.001)$ and weakly but still significantly with $\Delta$ force (FIGURE 1B), whereas neither GLS nor EF changes showed any correlation with coronary scores. An example of DSE with changes of 4 examined parameters in a patient with high values of SYNTAX and Gensini scores is presented in FIGURE3A and 3B.

In an attempt to exclude the impact of a myocardial scar on the GLS data, we limited subsequent analyses to a subgroup of 157 patients without a history of MI. In patients without MI, we found the strongest correlation with WMSI assessed at the peak stage of DSE (SYNTAX score, rho $=0.563$ and Gensini score, rho $=0.575 ; P<0.001$ for both scores). Similar values were recorded for correlation coefficients for $\Delta$ WMSI, see TABLE 5. In this group, the LV force at peak stress as well as $\Delta$ force also maintained a significant correlation with angiographic scores (TABLE 5). Contrarily, neither EF nor GLS showed any relationship with SYNTAX and Gensini scores at any stage of DSE in patients without MI history (FIGURE 1 C).

When limiting the analysis to 66 patients with a prior MI, WMSI maintained a correlation with the SYNTAX and Gensini scores at the peak DSE stage (Gensini score, rho $=0.402$ ) and for $\Delta$ values, but not for the rest (TABLE 6). Moreover, 
TABLE 1 Demographic characteristics, risk factors, and treatment of the study group

\begin{tabular}{ll} 
Parameter & Study group $(\mathbf{n}=223)$ \\
\hline Age, $y$, mean (SD); range & $62(9) ; 38-81$ \\
\hline Height, cm, mean (SD); range & $167(9) ; 146-187$ \\
\hline Body mass, kg, mean (SD); range & $81(15) ; 43-125$ \\
\hline Body mass index, kg/m², mean (SD); range & $28.7(4.7) ; 17.6-46.9$ \\
\hline Hypertension & $193(87)$ \\
\hline Diabetes mellitus & $58(26)$ \\
\hline Smoking & $128(57)$ \\
\hline Hypercholesterolemia & $197(88)$ \\
\hline Hypertriglyceridemia & $142(64)$ \\
\hline Family history of CAD & $39(18)$ \\
\hline History of MI & $66(30)$ \\
\hline Acetylsalicylic acid & $205(92)$ \\
\hline Clopidogrel & $69(31)$ \\
\hline B-Blockers & $178(80)$ \\
\hline Angiotensin-converting enzyme inhibitor & $182(82)$ \\
\hline Statin & $198(89)$ \\
\hline Long-acting nitrate & $112(50)$ \\
\hline
\end{tabular}

Data are presented as number (percentage) unless otherwise indicated.

Abbreviations: CAD, coronary artery disease; MI, myocardial infarction

TABLE 2 Comparison of parameters during dobutamine stress echocardiography in the study group $(n=223)$

\begin{tabular}{llll} 
Parameter & Mean (SD) at baseline & Mean (SD) at peak & $P$ value \\
Heart rate, bpm & $66(10)$ & $139(17)$ & $<0.001$ \\
\hline Systolic blood pressure, $\mathrm{mm} \mathrm{Hg}$ & $129(17)$ & $142(25)$ & $<0.001$ \\
\hline Diastolic blood pressure, $\mathrm{mm} \mathrm{Hg}$ & $71(10)$ & $77(16)$ & $<0.001$ \\
\hline EF, \% & $58(10)$ & $66(9)$ & $<0.001$ \\
\hline LV force, $\mathrm{mm} \mathrm{Hg} / \mathrm{ml}$ & $5.9(2.8)$ & $11.1(5.8)$ & $<0.001$ \\
\hline WMSI & $1.10(0.19)$ & $1.19(0.22)$ & $<0.001$ \\
\hline GLS, \% & $17.4(3.9)$ & $16.6(4.3)$ & $<0.001$
\end{tabular}

Abbreviations: EF, ejection fraction; GLS, global longitudinal strain in absolute values; LV, left ventricle; WMSI, wall motion score index

TABLE 3 Angiographic characteristics of patients with significant coronary lesions $(n=112)$

\begin{tabular}{llll} 
Lesion location & Single-vessel disease $(n=55)$ & 2-vessel disease $(n=35)$ & 3-vessel disease $(n=22)$ \\
\hline LMCA & 0 & 4 & 3 \\
\hline LAD & 19 & 29 & 22 \\
\hline CX & 22 & 19 & 22 \\
\hline RCA & 14 & 22 & 22
\end{tabular}

Abbreviations: CX, circumflex; LAD, left anterior descending; LMCA, left main coronary artery; RCA, right coronary artery 
TABLE 4 Correlations between SYNTAX and Gensini scores and echocardiographic indices of left ventricular function at baseline and during the peak stage of dobutamine stress echocardiography as well as with changes in the whole group $(n=223$ patients)

\begin{tabular}{|c|c|c|c|c|c|c|}
\hline \multirow[t]{2}{*}{ Parameter } & \multicolumn{2}{|c|}{ Rest } & \multicolumn{2}{|c|}{ Stress } & \multicolumn{2}{|c|}{$\Delta$} \\
\hline & rho & $P$ value & rho & $P$ value & rho & $P$ value \\
\hline \multicolumn{7}{|c|}{ SYNTAX score } \\
\hline $\mathrm{EF}$ & -0.129 & 0.06 & -0.149 & 0.03 & -0.017 & 0.81 \\
\hline GLS & $-0.236^{b}$ & $<0.001^{b}$ & -0.205 & 0.002 & -0.001 & 0.99 \\
\hline Force & -0.229 & $<0.001$ & $-0.346^{b}$ & $<0.001^{b}$ & $-0.242^{b}$ & $<0.001^{b}$ \\
\hline WMSI & $0.389^{a}$ & $<0.001^{\mathrm{a}}$ & $0.591^{\mathrm{a}}$ & $<0.001^{a}$ & $0.494^{a}$ & $<0.001^{a}$ \\
\hline \multicolumn{7}{|c|}{ Gensini score } \\
\hline $\mathrm{EF}$ & -0.164 & 0.02 & -0.191 & 0.006 & -0.009 & 0.89 \\
\hline GLS & $-0.263^{b}$ & $<0.001^{b}$ & -0.216 & 0.001 & 0.017 & 0.8 \\
\hline Force & -0.257 & $<0.001$ & $-0.377^{b}$ & $<0.001 b$ & $-0.261^{b}$ & $0.001^{b}$ \\
\hline WMSI & $0.407^{a}$ & $<0.001^{a}$ & $0.612^{\mathrm{a}}$ & $<0.001^{a}$ & $0.496^{a}$ & $<0.001^{a}$ \\
\hline
\end{tabular}

a Data for the variable showing the most potent correlation with angiographic scores

b The second variable in terms of the correlation strength

Abbreviations: $\Delta$ change calculated as differences between the peak and baseline values; others, see TABLE 2
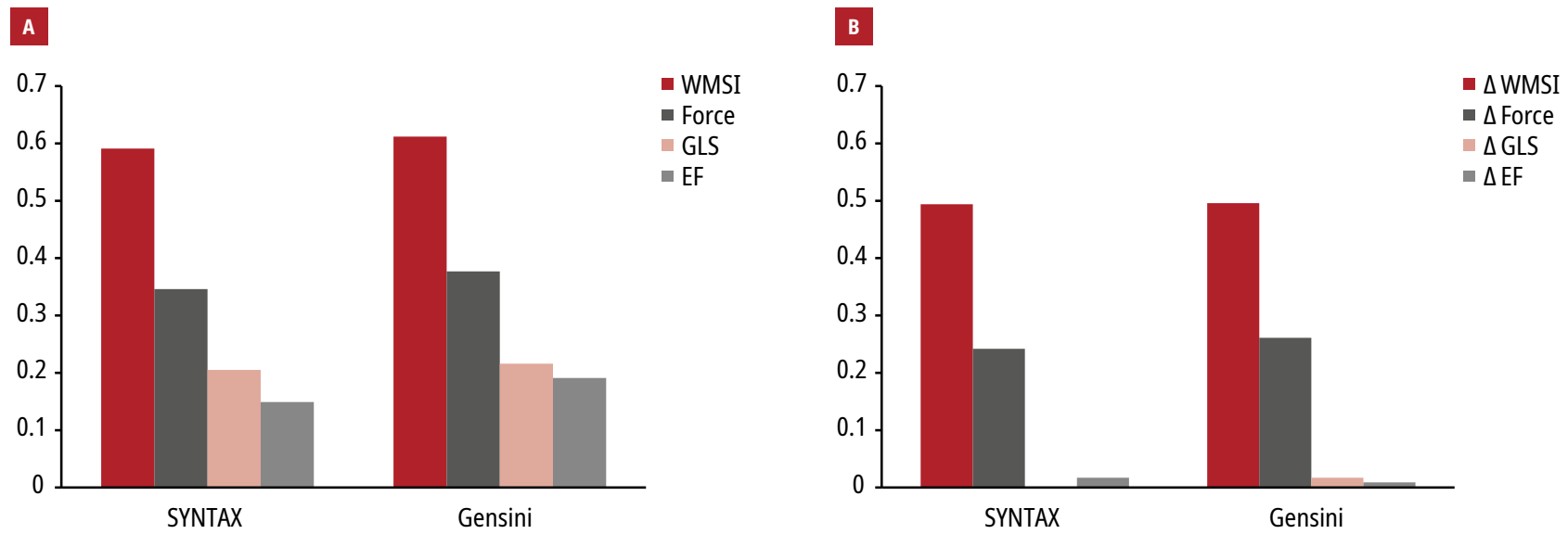

c
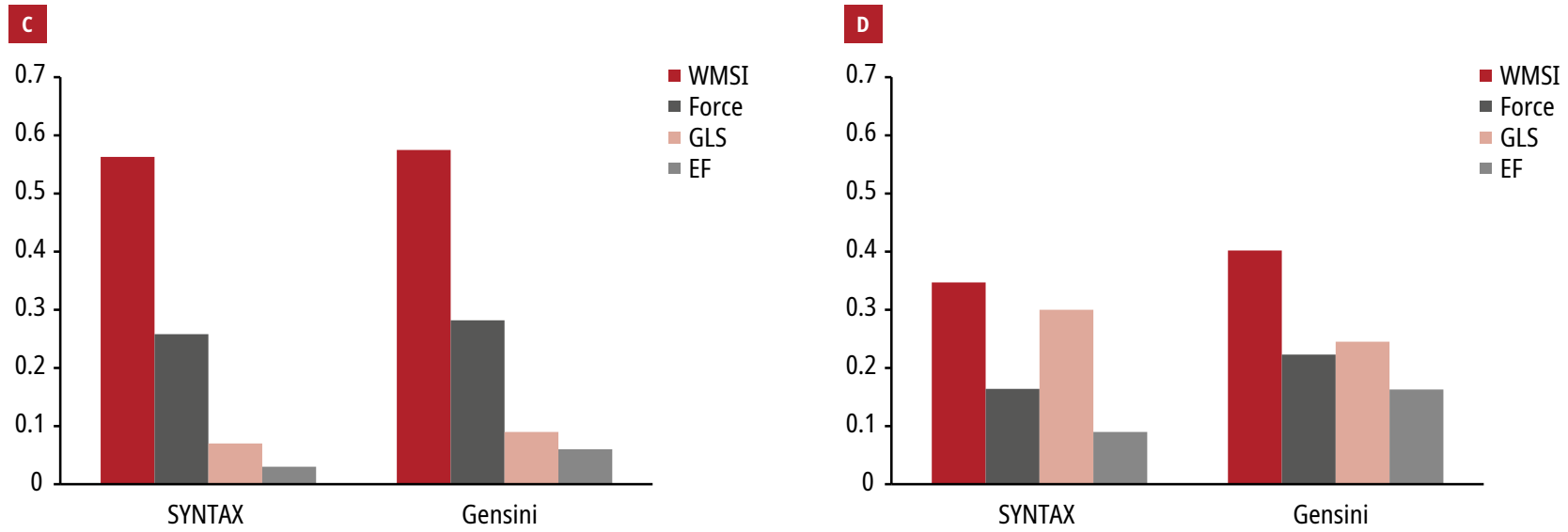

FIGURE 1 Comparisons of correlation strength between echocardiographic indices of left ventricle function (wall motion score index [WMSI], force, global longitudinal strain [GLS], and ejection fraction [EF]) and SYNTAX and Gensini scores. Y axis represents the values of correlation coefficient (rho) expressed in absolute numbers: $\mathbf{A}$ - correlation coefficients calculated at the peak stage of DSE versus SYNTAX and Gensini scores in the whole group $(n=223) ; \mathbf{B}-$ correlation coefficients calculated for changes $(\Delta)$ of indices between peak and baseline values during DSE versus SYNTAX and Gensini scores in the whole group $(n=223) ; \mathbf{C}-\operatorname{correlation}$ coefficients calculated at the peak stage of DSE versus SYNTAX and Gensini scores in the subgroup of patients without myocardial infarction $(n=157)$; D - correlation coefficients calculated at the peak stage of DSE versus SYNTAX and Gensini scores in the subgroup of patients with myocardial infarction $(n=66)$ 


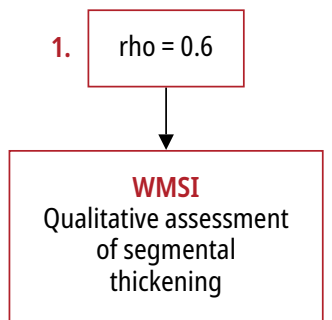

\begin{tabular}{|l|}
\hline \multicolumn{1}{|c|}{ WMSI } \\
\hline 1 \\
\hline $1.01-1.39$ \\
\hline $1.4-1.7$ \\
\hline$>1.7$ \\
\hline
\end{tabular}
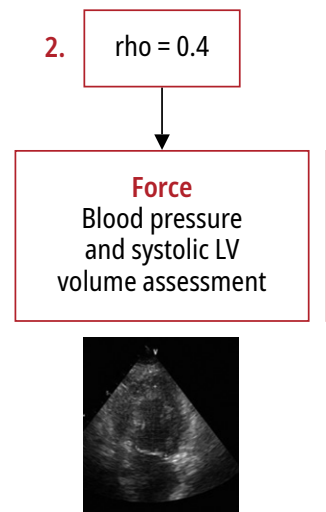
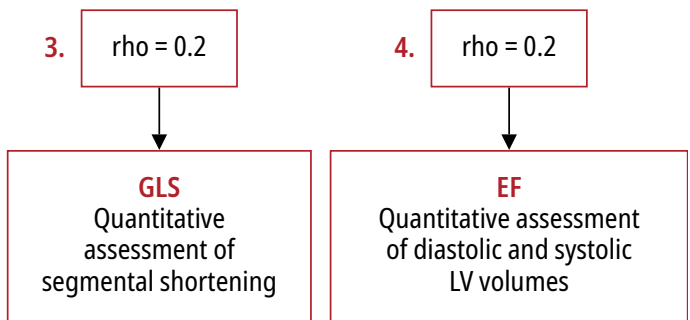

FIGURE 2 The hierarchy of echocardiographic parameters best predicting SYNTAX and Gensini scores at peak stage of dobutamine stress echocardiography. Rho represents a mean value of correlation coefficients for SYNTAX and Gensini scores and echocardiographic parameters.

Abbreviations: see TABLE2
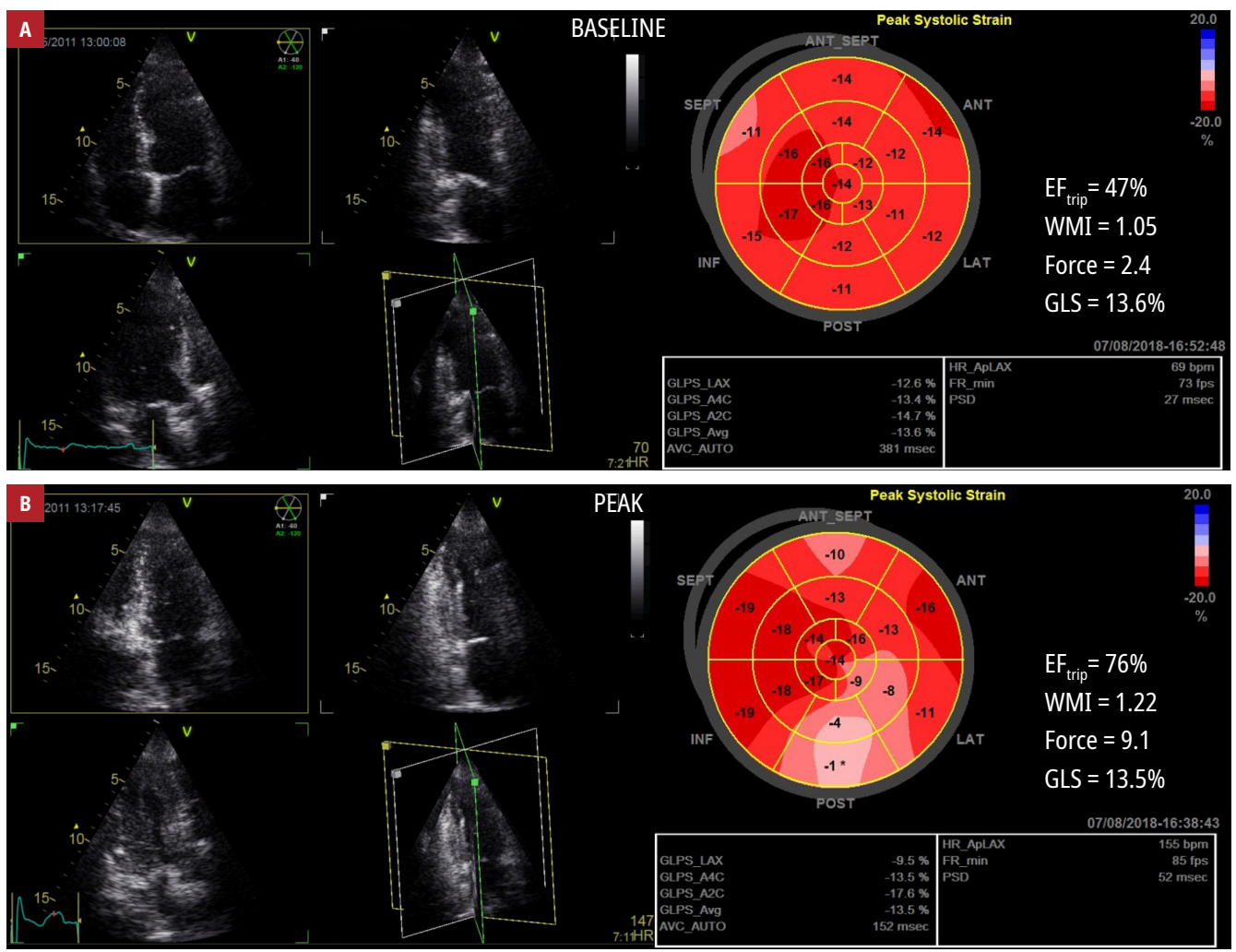

FIGURE 3 Dobutamine stress echocardiography at rest (A) and during peak stress (B) in a patient with high SYNTAX and Gensini scores (38 and 59, respectively). The abnormal response of wall motion score index (WMSI) was observed. Ejection fraction (EF) and force showed an increase and global longitudinal strain (GLS) remained constant despite some regions of impaired strain visible in the polar map, which, however, were counterbalanced by the increased strain of other segments. Abbreviations: $\mathrm{EF}_{\text {trip' }}$ triplane imaging

both angiographic scores showed a relationship with GLS at both stages of DSE (FIGURE 1D), with a maximal correlation coefficient observed for the SYNTAX score at the peak stage of DSE, with rho $=0.347$ and $P=0.004$, as shown in TABlE 6 .

DISCUSSION To the best of our knowledge, this is the first study to provide a direct comparison of 4 various indices of systolic LV function assessed at rest and during the peak stage of DSE in relation to underlying coronary artery disease severity. In a group of 223 patients (including those without significant CAD and with CAD, as well as those after MI, thus adequately representing patients undergoing SE in clinical practice) visual contractility assessment appeared as more closely related to CAD severity than quantitative parameters reflecting global LV function: force, GLS, and EF. 
TABLE 5 Correlations between SYNTAX and Gensini scores and echocardiographic indices of left ventricular function at baseline and during the peak stage of dobutamine stress echocardiography as well as with changes in the subgroup of patients without the history of myocardial infarction $(n=157)$

\begin{tabular}{|c|c|c|c|c|c|c|}
\hline \multirow[t]{2}{*}{ Parameter } & \multicolumn{2}{|c|}{ Rest } & \multicolumn{2}{|c|}{ Stress } & \multicolumn{2}{|c|}{$\Delta$} \\
\hline & rho & $P$ value & rho & $P$ value & rho & $P$ value \\
\hline \multicolumn{7}{|c|}{ SYNTAX score } \\
\hline $\mathrm{EF}$ & -0.03 & 0.7 & -0.03 & 0.72 & -0.018 & 0.83 \\
\hline GLS & -0.122 & 0.13 & -0.07 & 0.37 & 0.018 & 0.82 \\
\hline Force & -0.095 & 0.24 & $-0.258^{b}$ & $0.002^{b}$ & $-0.189^{b}$ & $0.02^{\mathrm{b}}$ \\
\hline WMSI & $0.214^{a}$ & $0.007^{a}$ & $0.563^{\mathrm{a}}$ & $<0.001^{a}$ & $0.590^{\mathrm{a}}$ & $<0.001^{\mathrm{a}}$ \\
\hline \multicolumn{7}{|c|}{ Gensini score } \\
\hline EF & -0.0534 & 0.51 & -0.0584 & 0.49 & -0.0138 & 0.87 \\
\hline GLS & -0.145 & 0.07 & -0.09 & 0.26 & 0.0151 & 0.85 \\
\hline Force & -0.107 & 0.19 & $-0.282^{b}$ & $<0.001^{b}$ & $-0.208^{b}$ & $0.01^{b}$ \\
\hline WMSI & $0.228^{a}$ & $0.004^{a}$ & $0.575^{a}$ & $<0.001^{a}$ & $0.598^{a}$ & $<0.001^{a}$ \\
\hline
\end{tabular}

a Data for the variable showing the most potent correlation with angiographic scores

b The second variable in terms of the correlation strength

Abbreviations: see TABLES 2 and 4

TABLE 6 Correlations between SYNTAX and Gensini scores and echocardiographic indices of left ventricular function at baseline and during the peak stage of dobutamine stress echocardiography as well as with changes in the subgroup of patients with the history of myocardial infarction $(n=66)$

\begin{tabular}{|c|c|c|c|c|c|c|}
\hline \multirow[t]{2}{*}{ Parameter } & \multicolumn{2}{|c|}{ Rest } & \multicolumn{2}{|c|}{ Stress } & \multicolumn{2}{|c|}{$\Delta$} \\
\hline & rho & $P$ value & rho & $P$ value & rho & $P$ value \\
\hline \multicolumn{7}{|c|}{ SYNTAX score } \\
\hline $\mathrm{EF}$ & -0.05 & 0.69 & -0.094 & 0.46 & -0.0577 & 0.65 \\
\hline GLS & $-0.253^{\mathrm{a}}$ & $0.04^{\mathrm{a}}$ & $-0.3^{b}$ & $0.01^{b}$ & -0.108 & 0.39 \\
\hline Force & -0.171 & 0.17 & -0.164 & 0.2 & -0.125 & 0.32 \\
\hline WMSI & 0.140 & 0.37 & $0.347^{a}$ & $0.004^{\mathrm{a}}$ & $0.294^{\mathrm{a}}$ & $0.02^{\mathrm{a}}$ \\
\hline \multicolumn{7}{|c|}{ Gensini score } \\
\hline $\mathrm{EF}$ & -0.126 & 0.32 & -0.163 & 0.2 & -0.036 & 0.78 \\
\hline GLS & $-0.259^{a}$ & $0.04^{\mathrm{a}}$ & $-0.245^{b}$ & $0.05^{b}$ & -0.01 & 0.94 \\
\hline Force & $-0.244^{b}$ & $0.048^{b}$ & -0.223 & 0.08 & -0.141 & 0.27 \\
\hline WMSI & 0.226 & 0.07 & $0.4^{\mathrm{a}}$ & $<0.001^{a}$ & $0.03^{a}$ & $0.03^{\mathrm{a}}$ \\
\hline
\end{tabular}

a Data for the variable showing the most potent correlation with angiographic scores

b The second variable in terms of the correlation strength

Abbreviations: see TABLES 1, 2, 3, and 4

Interestingly, WMSI was followed by force in the whole group as well as in patients without previous MI. Moreover, both parameters maintained their relationship with the severity of coronary lesions for their changes during DSE (FIGURE 1A-1C). It may indicate that mainly these 2 parameters have the potential to detect subtle changes of contractility caused by ischemia during SE, whereas the regional impairment of longitudinal shortening or segmental thickening is abolished or diminished by hypercontractility of nonischemic LV regions as rendered by GLS or EF. In addition, the compensatory hyperfunction of nonischemic zones does not affect the WMSI (normal segments = hyperkinetic segments $=1$ ) but may improve the value of EF, GLS, and force irrespective of the underlying CAD (an example is presented in FIGURE3Aand $3 \mathrm{~B}$ ).

In general, parameters measured during stress proved more valuable than those measured at rest or $\Delta$ indices, with the exception of GLS, which performed similarly at rest and during stress. 
As far as the correlation between regional longitudinal strain and visual contractility assessment is concerned, we observed in an earlier study a stepwise decrease of the absolute strain values in respective groups of segments with normo-, hipo-, and akinesis and this relationship was evident both at rest and during the stress stage of DSE. ${ }^{24}$ Nevertheless, baseline GLS did not correlate significantly neither with peak WMSI nor with $\Delta$ WMSI in patients without MI history, indicating that the potential for predicting the induced worsening of contractility on basis of the resting GLS data remains very limited.

On the other hand, GLS, while working quite well in the subgroup with a history of MI, does not predict the coronary score in patients without MI, which is a novel observation in comparison with limited data published so far (see FIGURE $1 C$ and 10 and TABLES 3,4 , and 5). ${ }^{25}$

One of the advantages of our study is the inclusion of both angiographic scores, SYNTAX and Gensini, which provided a kind of self-verification of the results. In our group the interobserver reproducibility was slightly higher for the SYNTAX score (kappa $=0.674)$ as compared with the study of Tanboga et al, ${ }^{26}$ and even better for the Gensini score (kappa $=0.840)$. We also found a low variability in WMSI between the experienced observers, as repeatedly shown in the past, when readers were exposed to joint reading sessions ignoring forms of questionable hypokinesis. ${ }^{27,28}$ The variability observed for GLS may appear less expected; however, during DSE with a high HR, suboptimal images may complicate data interpretation. ${ }^{29,30}$ Data regarding variability in segmental longitudinal strain at the baseline and peak stage of DSE in our patient group were published earlier, indicating greater impediments at the peak stage with an intraobserver variability of $16 \%$, as compared with $8.7 \%$ at rest. ${ }^{19,24}$

Currently, the comprehensive assessment of $\mathrm{LV}$, but also right ventricular function during stress echocardiography, is widely advocated in various clinical settings and numerous additional indices are tested, such as rotation, pulmonary pressures, or coronary flow reserve. ${ }^{31-35}$ It is worth to mention that both indices introduced in the present study, namely force and GLS, were based on a routine acquisition of apical views and did not prolong the time needed for the assessment of an individual patient (which in our group took <3 minutes for AFI analysis), which is in agreement with other studies. ${ }^{36,19}$

A more severe coronary stenosis is more likely to induce a deeper segmental alteration in the regional function (akinesia or dyskinesia) and generate a higher peak WMSI. Similarly, a more extensive CAD will induce a more diffuse alteration, also generating a higher WMSI. ${ }^{37}$

The following are the main clinical implications of our study: 1) evidence for a higher value of prevalence of visually assessed WMSI over quantitative parameters of global LV function in the prediction of underlying CAD; 2) presentation of the possible causes of inappropriateness / low sensitivity of peak GLS and EF as well as their changes to reflect the severity of underlying coronary disease which seems to be caused by hypercontractile response of nonischemic regions; 3) the stratification of LV function parameters during DSE according to their diagnostic utility for CAD severity assessed by appreciated SYNTAX and Gensini scores, and the observation indicating that force seems to be more closely related to the severity of CAD than most commonly used EF.

Limitations This is a single-center study, with a limited number of patients assessed while receiving full anti-ischemic therapy, which may protect from induced ischemia. ${ }^{38}$ However, this reflects also the contemporary population referred for SE. ${ }^{39,40}$

We used deformation analysis focusing only on longitudinal strain, which is, however, postulated to be the most suitable to detect subclinical LV dysfunction and the only one recommended by recent joint documents of the European Association of Cardiovascular Imaging and the American Society of Echocardiography for the evaluation of the LV function at rest and under stress conditions. ${ }^{12,13}$

Our results were obtained with DSE and may not necessarily apply to other forms of stress tests, which may further improve GLS analysis.

As to another limitation related to vascular assessment, it should be underlined that coronary angiography, even with SYNTAX and Gensini scores, assesses only lesion morphology and could be reinforced by functional estimation of stenosis with fractional flow reserve or coronary flow reserve during DSE. ${ }^{41}$

Finally, in the group without CAD lesions of $50 \%$ or more, we accepted the zero value for SYNTAX and Gensini scores, which meant that the marginal lesions, probably silent during DSE, were not taken into account in case of the Gensini score. ${ }^{42}$ Nevertheless, the simultaneous application of 2 angiographic scores strengthen the observed relationships.

Conclusions Peak WMSI and, to a lesser extent, peak force are more useful than EF and GLS to reflect the angiographic extent of CAD with DSE in patients with and without previous MI. GLS correlates only weakly with coronary scores in unselected patients as well as in patients after MI at rest and at peak stress, and not at all in patients without MI. Peak WMSI is less time consuming and does not require additional software and analysis time as compared with EF, force, and GLS. It emphasizes the importance of the human factor (visual assessment 
of regional contractility performed by an experienced observer) in providing clinically and prognostically relevant DSE results.

Nowadays, we still need to analyze and understand both the advantages and inherent limitations offered by deformation assessment and other advanced techniques to be able to incorporate them accurately into DSE, since without a full understanding, less is more in the SE assessment, and will remain as such for a long time.

\section{ARTICLE INFORMATION}

ACKNOWLEDGMENTS This work was partially supported by a grant from the State Committee for Scientific Research, (no. NN402500240; to KW-D and JDK). CONFLICT OF INTEREST None declared.

OPEN ACCESS This is an Open Access article distributed under the terms of the Creative Commons Attribution-Non Commercial-No Derivatives $4.0 \mathrm{In}$ ternational License (CC BY-NC-ND 4.0), allowing third parties to download articles and share them with others, provided the original work is properly cited, not changed in any way, distributed under the same license, and used for noncommercial purposes only. For commercial use, please contact the journal office at kardiologiapolska@ptkardio.pl.

HOW TO CITE Wierzbowska-Drabik K, Picano E, Simiera M, et al. A head-to-head comparison of wall motion score index, force, strain, and ejection fraction for the prediction of SYNTAX and Gensini coronary scores by dobutamine stress echocardiography. Kardiol Pol. 2020; 78: 715-724. doi:10.33963/KP.15376

\section{REFERENCES}

1 Sianos G, Morel MA, Kappetein AP, et al. The SYNTAX score: an angiographic tool grading the complexity of coronary artery disease. EuroIntervention. 2005; 1:219-227.

2 Gensini GGMD. The pathological anatomy of the coronary arteries of man. In: Gensini GGMD, ed. Coronary Arteriography. Futura Publishing Co; 1975: 271-274.

3 Palmerini T, Genereux P, Caixeta A, et al. Prognostic value of the SYNTAX score in patients with acute coronary syndromes undergoing percutaneous coronary intervention: analysis from the ACUITY (Acute Catheterization and Urgent Intervention Triage StrategY) trial. J Am Coll Cardiol. 2011; 57: 2389-2397.

4 Gao G, Feng L, Zhang D, et al. Proximal left anterior descending coronary artery stenosis should be considered when using the prognostic value of the residual SYNTAX score: data from 10343 consecutive patients with long-term follow up in the real world. Catheter Cardiovasc Interv. 2018; 91: 639-645.

5 Witberg G, Zusman 0 , Codner $P$, et al. Impact of coronary artery revascularization completeness on outcomes of patients with coronary artery disease undergoing transcatheter aortic valve replacement: a meta-analysis of studies using the residual SYNTAX score (Synergy Between PCI With Taxus and Cardiac Surgery). Circ Cardiovasc Interv. 2018; 11: e006000.

6 Zencirci AE, Zencirci E, Degirmencioglu A, et al. The relationship between Gensini score and ST-segment resolution in patients with acute ST-segment elevation myocardial infarction undergoing primary percutaneous coronary intervention. Kardiol Pol. 2014; 72: 494-503.

7 Marwick T, D'Hondt AM, Baudhuin T, et al. Optimal use of dobutamine stress for the detection and evaluation of coronary artery disease: combination with echocardiography or scintigraphy or both? J Am Coll Cardiol. 1993; 22: 159-167.

8 Picano E, Alaimo A, Chubuchny V, et al. Noninvasive pacemaker stress echocardiography for diagnosis of coronary artery disease: a multicenter study. J Am Coll Cardiol. 2002; 40: 1305-1310.

9 Montalescot G, Sechtem U, Achenbach S, et al. 2013 ESC guidelines on the management of stable coronary artery disease: the Task Force on the management of stable coronary artery disease of the European Society of Cardiology. Eur Heart J. 2013; 34: 2949-3003.

10 Pellikka PA, Nagueh SF, Elhendy AA, et al; American Society of Echocardiography. American Society of Echocardiography recommendations for performance interpretation, and application of stress echocardiography. J Am Soc Echocardiogr. 2007; 20: 1021-1041

11 Sicari R. Risk stratification by stress echocardiography beyond wall motion analysis. JACC: Cardiovascular Imaging 2009; 2: 260-262.

12 Lancellotti P, Pellikka PA, Budts W, et al. The clinical use of stress echocardiography in non-ischaemic heart disease: recommendations from the European Association of Cardiovascular Imaging and the American Society of Echocardiography. J Am Soc Echocardiogr. 2017; 30: 101-138.

13 Galderisi M, Cosyns B, Edvardsen T, et al. Standardization of adult transthoracic echocardiography reporting in agreement with recent chamber quantification, diastolic function, and heart valve disease recommendations: an expert consensus document of the European Association of Cardiovascular Imaging. Eur Heart J Cardiovasc Imaging. 2017; 18: 1301-1310.

14 Ginzton LE, Laks MM, Brizendine $M$, et al. Noninvasive measurement of the rest and exercise peak systolic pressure/end-systolic volume ratio: a sensitive two-dimensional echocardiographic indicator of left ventricular function. J Am Coll Cardiol. 1984; 4: 509-516.

15 Bombardini T. Myocardial contractility in the echo lab: molecular, cellula and pathophysiological basis. Cardiovasc Ultrasound. 2005; 3: 27.

16 Bombardini T, Zoppè M, Ciampi Q, et al. Myocardial contractility in the stress echo lab: from pathophysiological toy to clinical tool. Cardiovasc Ultrasound. 2013; 11:41.

17 Grosu A, Bombardini T, Senni M, et al. End-systolic pressure/volume relationship during dobutamine stress echo: a prognostically useful non-invasive index of left ventricular contractility. Eur Heart J. 2005; 26: 2404-2412.

18 Cortigiani L, Huqi A, Ciampi Q, et al. Integration of wall motion, coronary flow velocity, and left ventricular contractile reserve in a single test: prognostic value of vasodilator stress echocardiography in patients with diabetes. I Am Soc Echocardiogr. 2018; 31: 692-701.

19 Wierzbowska-Drabik K, Hamala P, Roszczyk N, et al. Feasibility and correlation of standard 2D speckle tracking echocardiography and automated function imaging derived parameters of left ventricular function during dobutamine stress test. Int J Cardiovasc Imaging. 2014; 30: 729-737.

20 Wierzbowska-Drabik K, Hamala P, Kasprzak JD. Delayed longitudinal myocardial function recovery after dobutamine challenge as a novel presentation of myocardial dysfunction in type 2 diabetic patients without angiographic coronary artery disease. Eur Heart J Cardiovasc Imaging. 2015; 16: 676-683.

21 Lang RM, Badano LP, Mor-Avi V, et al. Recommendations for cardiac chamber quantification by echocardiography in adults: an update from the American Society of Echocardiography and the European Association of Cardiovascular Imaging. J Am Soc Echocardiogr. 2015; 28: 1-39.

22 Sicari R, Nihoyannopoulos P, Evangelista A, et al. Stress echocardiography expert consensus statement - executive summary. Eur Heart J. 2009; 30: 278-289.

23 Pellikka PA, Nagueh SF, Elhendy AA, et al. American Society of Echocardiography recommendations for performance, interpretation, and application of stress echocardiography. J Am Soc Echocardiogr. 2007; 20: 1021-1041.

24 Wierzbowska-Drabik K, Miśkowiec D, Kasprzak JD. Systolic longitudinal strain correlates with visual assessment of regional left ventricular function during dobutamine stress echocardiography and discriminates the segments with induced contractility impairment. Echocardiography. 2017; 34: 1020-1028.

25 Vrettos A, Dawson D, Grigoratos C, Nihoyannopoulos P. Correlation between global longitudinal peak systolic strain and coronary artery disease severity as assessed by the angiographically derived SYNTAX score. Echo Res Pract. 2016; 3: 29-34. 26 Tanboga I, Ekinci M, Isik T, et al. Reproducibility of syntax score: from core lab to real world. J Interven Cardiol. 2011; 24: 302-306.

27 Picano E, Lattanzi F, Orlandini A, et al. Stress echocardiography and the human factor: the importance of being expert. J Am Coll Cardiol. 1991; 17: 666-669.

28 Varga A, Picano E, Dodi C, et al. Madness and method in stress echo reading. Eur Heart]. 1999; 20: 1271-1275.

29 Hubbard RT, Arciniegas Calle MC, Barros-Gomes S, et al. 2-dimensional speckle tracking echocardiography predicts severe coronary artery disease in women with normal left ventricular function: a case-control study. BMC Cardiovasc Disord. 2017; 17: 231 .

30 Smiseth $\mathrm{OA}$, Torp H, Opdahl A, et al. Myocardial strain imaging: how useful is it in clinical decision making? Eur Heart J. 2016; 37: 1196-1207.

31 Agoston G, Morvai-Illes B, Palinkas A, Varga A. The role of stress echocardiography in cardiovascular disorders. Kardiol Pol. 2019; 77: 1011-1019.

32 Mielczarek A, Kasprzak JD, Lipiec $P$, et al. Quantitative assessment of the rotation and twist of the left ventricle during dobutamine stress echocardiography: a comparison of patients with and without significant coronary artery disease. Kardiol Pol. 2019; 77: 696-702.

33 Wierzbowska-Drabik K, Picano E, Bossone E, et al. The feasibility and clinical implication of tricuspid regurgitant velocity and pulmonary flow acceleration time evaluation for pulmonary pressure assessment during exercise stress echocardiography. Eur Heart J Cardiovasc Imaging. 2019; 20: 1027-1034.

34 Ferrara F, Zhou X, Gargani L, et al. Echocardiography in pulmonary arterial hypertension. Curr Cardiol Rep. 2019; 21: 22.

35 Ciampi Q, Zagatina A, Cortigiani L, et al. Functional, anatomical, and prognostic correlates of coronary flow velocity reserve during stress echocardiography. J Am Coll Cardiol. 2019; 74: 2278-2291.

36 Picano E, Bombardini T, Kovačević Preradović T, et al. Left ventricular contractile reserve in stress echocardiography: the bright side of the force. Kardiol Pol. 2019; 77: 164-172.

37 Lu C, Picano E, Pingitore A, et al. Complex coronary artery lesion morphology influences results of stress echocardiography. Circulation. 1995; 91: 1669-1675.

38 Sicari R, Cortigiani L, Bigi R, et al; Echo-Persantine International Cooperative (EPIC) Study Group; Echo-Dobutamine International Cooperative (EDIC) Study Group. Prognostic value of pharmacological stress echocardiography is affected by concomitant antiischemic therapy at the time of testing. Circulation. 2004; 109: 2428-2431.

39 Papachristidis A, Roper D, Cassar Demarco D, et al. The prognostic role of stress echocardiography in a contemporary population and the clinical significance of limited apical ischaemia. Echo Res Pract. 2016; 3: 105-113. 
40 Carpeggiani C, Landi P, Michelassi C, et al. The declining frequency of inducible myocardial ischemia during stress echocardiography over 27 consecutive years (1983-2009). Int J Cardiol. 2016; 224: 57-61.

41 Picano E, Ciampi Q, Wierzbowska-Drabik K, et al. The new clinical standard of integrated quadruple stress echocardiography with $A B C D$ protocol. Cardiovasc Ultrasound. 2018; 16: 22.

42 Sinning C, Zengin E, Waldeyer C, et al. SYNTAX score-0 patients: risk stratification in nonobstructive coronary artery disease. Clin Res Cardiol. 2016; 105: 901-911. 